\title{
Looming flu pandemic has experts crying fowl
}

The death last month of a Hong Kong man infected with $\mathrm{H} 5 \mathrm{~N} 1$, an avian influenza virus, has once again sounded the alarm for a global influenza pandemic. In 1997, the same virus killed six people, in each case by direct transmission from chickens. The virus has not yet acquired the ability to spread from person to person but many experts say it is only a matter of time.

"We've had H5N1 since 1997, we don't have a vaccine on the shelf. That is a black eye for [the World Health Organization] and the system," says Robert Webster, an influenza expert at Saint Jude's Children's Hospital in Memphis, Tennessee. "What the hell have we been doing?"

At least in some corners of the world, people are finally paying attention. In May, the WHO is expected to pass a resolution urging member states to prepare for the worst; the journal Vaccine is dedicating its May issue to research on influenza; and human safety tests for a recombinant vaccine against $\mathrm{H} 5 \mathrm{~N} 1$ are expected to begin this summer.

Those preparations may be just in the nick of time: as Nature Medicine went to press, the US state of Connecticut was planning to vaccinate millions of chickens when birds at the state's largest egg farm became infected with avian influenza. Worldwide, health officials also warned that a mysterious respiratory illness that had thus far claimed the lives of nine people could be a new strain of flu.

The most devastating pandemic of the last century was the 'Spanish Flu' of 1918, which is estimated to have killed up to 50 million people worldwide. Milder pandemics in 1957 and 1968 each killed

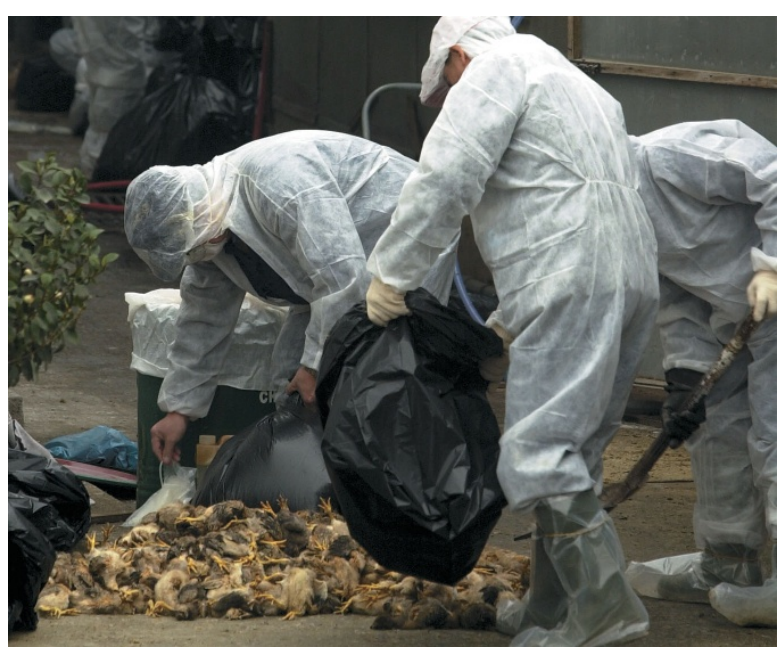

Count your chickens: Hong Kong health workers slaughtered 1.4 million birds during the 1997 influenza outbreak around 1 million people. All three involved strains of influenza virus originally derived from birds, the natural reservoir for the virus, with some genes from strains that infect humans. The Hong Kong strain, H5N1, is named for the surface antigens hemagglutinin $(\mathrm{H})$ and neuraminidase (N).

Webster and others are using reverse genetics to create hybrid viruses with any 1 of the 15 different varieties of hemagglutinin genes and 9 different neuraminidase genes stitched onto a human influenza virus backbone. Researchers hope to create 'seed stocks' of the engineered flu viruses that could be pulled off the shelf to produce large quantities of vaccine as soon as a pandemic strain is identified.

Reverse genetics is the only option, according to Webster. "There is no alternative strategy at this time to rapidly make a vaccine."

Kanta Subbarao, a researcher at the US National Institutes of Health, will soon publish the first successful attempt to generate an H5N1-like recombinant vaccine candidate using reverse genetics. In mice, the vaccine is safe and protects against challenge with wild-type H5N1 virus. Human safety and immunogenicity tests will begin this summer, Subbarao says. Because of recent events, there's an added urgency, she says. "But there's very little experience in terms of what it would take to immunize somebody against the avian virus."

The WHO resolution aims to increase routine vaccination of all people at high risk-including at least $50 \%$ of the elderly population-by 2006. It also points to the need to control annual epidemics that kill around a million people a year worldwide.

Arnold Monto, professor of epidemiology at the University of Michigan School of Public Health, welcomes the move. "This is the first time [influenza has been a high priority]," he says. "Pandemics tend to be the lever that gets people's attention."

Vaccines now have nearly $90 \%$ efficacy in healthy adults. But only 250 million doses are distributed each year, catering to less than $5 \%$ of the world's population. Developing a vaccine is also just one piece of the puzzle. Fewer than 30 countries have planned for a pandemic, and "only a handful" have translated this plan into concrete policies and action, says Nancy Cox, director of the influenza branch at the Centers for Disease Control and Prevention.

Many developing countries lack influenza vaccination policies altogether because they have no data on the disease burden to compare with other major diseases such as AIDS, tuberculosis and malaria. Recent outbreaks show how destructive influenza can be in such nations. In the Democratic Republic of Congo, an influenza epidemic has now infected more than 1.5 million people, and killed more than 2,000, while an outbreak in Madagascar last year killed more than 800 .

Even under the best of circumstances, it is not easy to protect the most vulnerable. In the developed world, for instance, greater life expectancy means an increasing population of the elderly, who are more vulnerable to influenza. In January, the Journal of the American Medical Association reported that the number of elderly people dying from influenza has tripled in the past three decades. The vaccine may also be less effective in the elderly.

"We don't yet have a good understanding of how to improve immunogenicity in the elderly and deal with the features of an aging immune system," says Maria Zambon of the UK's Public Health Laboratory Service. "You're not going to make, in my view, a new universal panacea for influenza."

Julie Clayton, London 